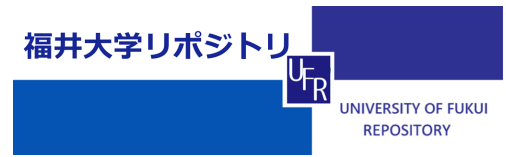

Synt hesi s and the el ectrol yte of the f unct i onal pol yst yr en-based I at ex

\begin{tabular}{|l|l|}
\hline $\begin{array}{l}\text { jour nal or } \\
\text { publ i cat i on titl e }\end{array}$ & $214 \mathrm{t}$ Neet ing of the El ect rochemical Soci ety \\
\hline page range & 139 \\
\hline year & 2008 10 \\
\hline URL & ht t p: //hdl . handl e. net /10098/5210 \\
\hline
\end{tabular}




\section{Synthesis and the electrolyte of the functional polystyren-based latex}

Tomoyasu Sakata, Koichi Aoki, Jingyuan Chan

Department of Applied Physics, University of Fukui,

3-9-1 Bunkyo, Fukui-shi, 910-8507 Japan

Occurrence of electrode reactions requires formation of an electric double layer at the electrode surface.

Although a distribution of ions in a double layer can be calculated from the Gouy-Chapman equation, it has not been able to prove the validity. It is desirable to see the formation and the structure of diffuse double layers. Visibly sized ionic particle, ionic latex particles, are expected to allow us to visualize the formation and the structure of the double layer. Our aim is to see the motion of visible large molecules by use of the latex which has strongly acid substitution, styrene sulfonate. The latex is predicted to work for supporting electrolyte and formation of the charge double layer at the electrode.

The latex, polystyrene-polystyrene sulfonate, was synthesized from polymerization of the styrene and then copolymerization of styrene sulfonate, according to the previous method ${ }^{1}$. Styrene was purified by distillation. Poly( $N$-vinylpyrrolidone)(PVP) was used as a stabilizer or surfactant during the dispersion polymerization of styrene. $\alpha$-azoisobutyronitrile(AIBN), 2-propanol, and the PVP were used as received. PVP was dissolved in 2propanol in a three-necked round-bottomed flask. The mixture maintained to $70{ }^{\circ} \mathrm{C}$ under the nitrogen atmosphere for $12 \mathrm{~h}$. A solution of styrene containing AIBN was added drop-wise, and then methanol and sodium styrene sulfonate were added drop-wise to vigorously stirred PVP/2-propanol solution for $24 \mathrm{~h}$. The solution became a milky white suspension. The suspension had optically visible particles (Fig.1) which polymerized was dispersed uniformity in water.

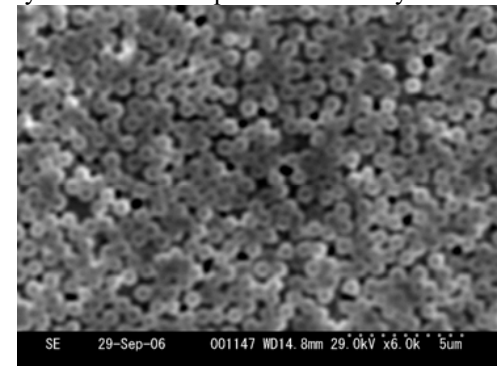

Fig.1.SEM Photograph of polystyrene-polystyrene sulfonate.

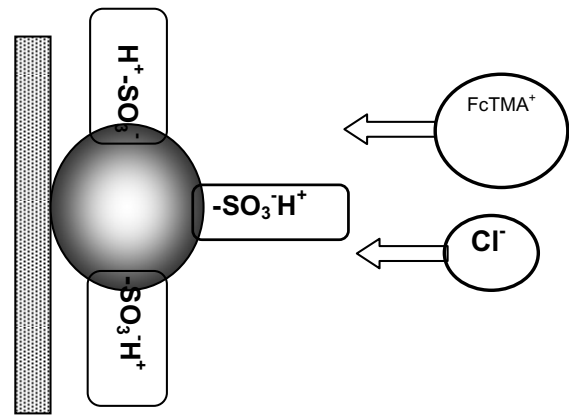

(A)
(B) ${ }^{1}$ C.Wang, T Nagahashi, K.Aoki, J.Chen, J.Electroanal

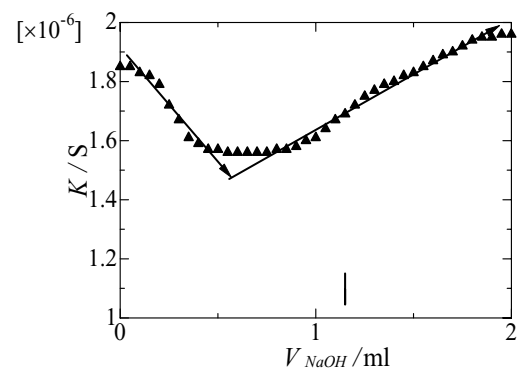

Fig.2. Variation of conductance of the latex suspension when $\mathrm{NaOH}$ solution was titrated.

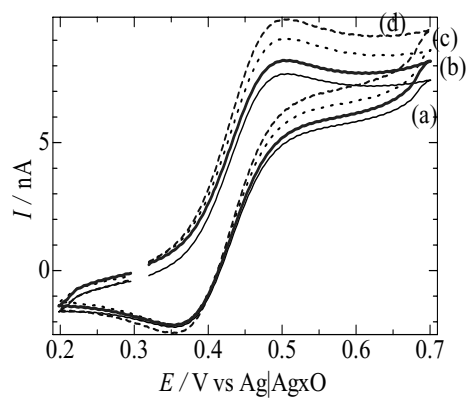

Fig.3. Cyclic voltammograms of FcTMA-including polystyrene sulfonate suspensions for (a) FcTMA 1mM, (b) with latex $0.004 \mathrm{mM}$, (c) 1.32 with latex $0.01 \mathrm{mM}$, (d) with latex $0.02 \mathrm{mM}$.

Concentration of sulfonate per particle evaluated by conductometric titration (Fig.2) was $6.5 \mathrm{mM}$, suggesting a strong acid. Cyclic voltammetry was made in the latex suspension including (ferrocenylmethyl) trimethylammonium chloride (FcTMA) and sodium ferrococenylsulfonate (Fig.3). The anodic current peak increased as concentration of the latex was higher. The increase can be explained in terms of electric migration, as follows. $\mathrm{FcTMA}^{+}$is ionically associated with $\mathrm{Cl}^{-}$in the bulk, as sulfonate latex is with $\mathrm{H}^{+}$, because salt is absent in the bulk. When FcTMA ${ }^{+}$diffuses to the electrode, $\mathrm{Cl}$ necessarily diffuses together in order to keep electric neutrality. Then the diffusional flux is twice (Fig. 4(A)) the flux in the presence of salt (Fig.4(B)).

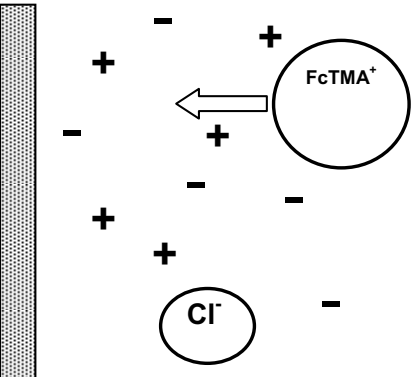
Chem.,530(2002) 47 .

Fig.4.Illustration of ion transfer in solution for (A) without and (B) with salt. (A) contains sulfonate latex. 